\title{
GEOMETRIC CONDITIONS ON THE TYPE OF MATTER DETERMINING THE FLAT BEHAVIOR OF THE ROTATIONAL CURVES IN GALAXIES
}

\author{
Tonatiuh Matos $^{a}$, Darío Núñez ${ }^{b}$, F. Siddhartha Guzmán ${ }^{a}$, Erandy Ramírez ${ }^{b}$ \\ ${ }^{a}$ Departamento de Física, \\ Centro de Investigación y de Estudios Avanzados del I. P. N., \\ A. P. 14-700, 07000 México, D.F.,MEXICO \\ ${ }^{b}$ Instituto de Ciencias Nucleares, \\ Universidad Nacional Autónoma de México \\ A. P. 70-543, 04510 México, D. F., MEXICO
}

(October 29, 2018)

\begin{abstract}
In an arbitrary axisymmetric stationary spacetime, we determine the expression for the tangential velocity of test objects following a circular stable geodesic motion in the equatorial plane, as function of the metric coefficients. Next, we impose the condition, observed in large samples of disks galaxies, that the magnitude of such tangential velocity be radii independent, obtaining a constraint equation among the metric coefficients, and thus arriving to an iff condition: The tangential velocity of test particles is radii independent iff the metric coefficients satisfied the mentioned constraint equation. Furthermore, for the static case, the constraint equation can be easily integrated, leaving the spacetime at the equatorial plane essentially with only one independent metric coefficient. With the geometry thus fixed, we compute the Einstein tensor and equate it to and arbitrary stress energy tensor, in order to determined the type of energy-matter which could produce such a geometry. Within an approximation, we deduce a constraint equation among the components of the stress energy tensor. We test in that constraint equation several well known types of matter, which have been proposed as dark matter candidates and are able to point for possible right ones. Finally, we also present the spherically symmetric static case and apply the mentioned procedure to perfect fluid stress energy tensor, recovering the Newtonian result as well as the one obtained in the axisymmetric case. We also present arguments on the need to use GR to study types of matter different than the dust one.
\end{abstract}

PACS numbers: 95.35.+d, 95.35.G

\section{INTRODUCTION}

One of the most important achievements of the present cosmology is doubtless the complete identifications and the accounting of the amounts of the different types of matter and energy which are present in the Universe (for an excellent review see for example [1]). Essentially, the present components of the Universe are composed by matter and vacuum energy $\Omega_{0}=\Omega_{M}+\Omega_{\Lambda}$ [2]. Furthermore, there is a very good evidence that the Universe is flat. That evidence comes either from the theory, where the most accepted model for the early Universe is inflation, as well as from observational data, which implies that $\Omega_{0}=1 \pm 0.12$ (see for example [3]). The mass of the galaxy clusters is perhaps the most reliable way for determining the matter component $\Omega_{M}$. Observations indicate that $\Omega_{M} \sim 0.3$ [西, however, the main visible components of $\Omega_{M}$, baryons, neutrinos, form a very small fraction of $\Omega_{M}$. Observations indicate that stars and dust (baryons) represents something like $5 \%$ of the whole matter of the Universe. In other words, $\Omega_{M} \sim \Omega_{b}+\Omega_{D M} \sim 0.05+\Omega_{D M}$, where $\Omega_{D M}$ represents the dark matter part of the matter contributions which has to have a value of $\Omega_{D M} \sim 0.25$. Notice that the given value of the amount of baryonic matter is in concordance with the limits imposed by nucleosynthesis (see for example [1]). A greater amount of baryonic matter could change the predicted values of primordial $\mathrm{H}$ and ${ }^{4} \mathrm{He}$ in the standard model of cosmology which coincide very well with observations.

The existence of dark matter in the Universe has been firmly established by astronomical observations at very different length-scales, ranging from the local galaxies to clusters of galaxies. The standard way to notice this need for dark matter comes within the framework of mechanics: A large fraction of the mass needed to produce the observed dynamical effects in all these very different systems, is not seen. This puzzle has stimulated the exploration of several proposals, and very imaginative explanations have been put forward, from exotic matter like supersymmetric particles [5] to non relativistic modifications of Newtonian dynamics [6] and non-linear general relativistic theories [7]. Above this, it is believed that this dark matter is such that interacts very weakly with ordinary matter, which makes it very difficult to detect by other means other than by their gravitational effects on the baryonic matter as it is well 
stablished for the cold dark matter scenario [8.97. The bottom line is that one of the most important components of the density of the Universe, the dark matter, still eludes us, and the question remains open: Which is the nature of the dark matter component?

At the galactic scale, the problem is clearly posed: The measurements of rotation curves (RC) in galaxies show that the coplanar orbital motion of gas in the outer parts of these galaxies keeps a more or less constant velocity up to several luminous radii [10,11]. The discrepancy arises when one applies the usual Newtonian dynamics to the observed luminous matter and gas, since then, the circular velocity should decrease as we move outwards. The most widely accepted explanation is that there exists a spherical halo of dark matter, its nature being unknown, which surrounds the galaxy and account for the missing mass needed to produce the flat behavior of the RC.

The main goal of this work is to study the dark matter problem in spiral galaxies, using a fully relativistic approach, continuing with the work started by Matos and Guzmán [12], where they made a preliminary dynamical analysis in the context of spiral galaxies. We apply a deductive method: Starting from a reasonable general space-time, we deduce, in terms of arbitrary metric coefficients the expression for the tangential velocity of test objects following a circular stable geodesic motion in the equatorial plane. We impose next the condition, observed in hundreds of galaxies, that such tangential velocity be radii independent, and obtain a constraint equation among the metric coefficients, Eq.(25). Arriving in this way to an iff condition: In an axisymmetric static space-time, the tangential velocity of test particles at the equatorial plane is radii independent iff the metric coefficients satisfied Eq.(25). Furthermore, for the static case, the constraint equation can be solved, leaving the space-time essentially with only one independent metric coefficient, thus determining very narrowly the type of space-time which can have a geometry such that the tangential velocity of the geodesics of test particles moving in stable circular equatorial orbits, be radii independent. With the geometry thus fixed, we compute the Einstein tensor and equate it to and arbitrary stress energy tensor, in order to determined the type of energy-matter which could produce such a geometry. We are able to deduce a constraint equation among the components of the stress energy tensor, Eq.(47). Using that constraint equation, Eq.(47), we test several well known types of matter, which have been proposed as dark matter candidates and are able to impose further restrictions on most of them as possible candidates for the dark matter in the region where it is observed the mentioned behavior on the tangential velocity. It is stimulating that the gravitational physics has been developed to such degree in which we can actually follow Sherlock Holmes' maxim: "...when you have excluded the impossible, whatever remains, however improbable, must be the truth" 13.

The work is composed as follows: In section 2 we determine the geometry of axisymmetric static space times allowing a tangential velocity with a magnitude independent of the radius. In section 3, we work with the Einstein equations for the geometry thus determined and for a general stress energy tensor, obtaining a constraint for the components of such stress energy tensor, and we test four types of matter into that constraint, being able to restrict most of them, among which is the perfect fluid. Finally, in the conclusion we discuss our results and propose a coupling of types of matter, which could be the one we are looking for in order to determine the nature of the dark matter. We also present this analysis for the spherical static space time in appendix $\mathrm{V}$ and for the axisymmetric stationary case in appendix V.

\section{FORM OF THE LINE ELEMENT}

In this section we study the conditions which the flatness of the tangential velocity of the RC, imposes on the metric coefficients. We want to stress the fact that the results presented in this section are independent of type of energy-matter tensor present in the space-time and curving it. It is a purely geometric analysis.

As mentioned above, observational data show that the galaxies are composed by almost $90 \%$ of dark matter. Thus we can suppose that luminous matter does not contribute in a determining way to the total energy density of the galaxy, at least in the region where the flatness of the $\mathrm{RC}$ is observed. Consequently, we consider that the dark matter will be the main contributor to the dynamics, and we will treat the observed luminous matter as a test fluid, that's it, in this approximation we will neglect the contribution of the luminous matter to the curvature, $i$. e., to the dynamics. Also, it is reasonable to suppose that the halo of dark matter is symmetric with respect to the rotation axis of the galaxy, thus we take the symmetry of the space-time as axially symmetric. Furthermore, the observations allow us to take the space-time as stationary as well. Thus, the most general reasonable space-time which we can study is an axisymmetric stationary one. The line-element of such space-time, given in the Papapetrou form is: 


$$
d s^{2}=-e^{2 \psi}(d t+\omega d \varphi)^{2}+e^{-2 \psi}\left[e^{2 \gamma}\left(d \rho^{2}+d z^{2}\right)+\mu^{2} d \varphi^{2}\right]
$$

where $\psi, \omega, \gamma$, and $\mu$, are functions of $(\rho, z)$.

We will derive the geodesic equations in the equatorial plane, that is for $z=\dot{z}=0$, where dot stands for the derivative with respect to the proper time, $\tau$. Then, we will study the constrains imposed for circular geodesics on the energy and angular momentum of particles in such orbits, and obtain an expression for the tangential velocity for the particles moving along those geodesics, described in terms of the metric coefficients. Finally, we impose the condition that such tangential velocity be radii independent and derive the restriction that then has to be satisfied among the gravitational coefficients. In this section we derive the expressions for a static space time and in the Appendix $\square$ we do so for the stationary one. We split the presentation in this way not only for the sake of clarity, but from the fact that the static case is also quite realistic, considering that the observed velocity of the stars orbiting a galaxy in the region of interest is quite non relativistic (of the order of $230 \mathrm{Km}$ per s), thus we can infer that the space-time is not very rapidly rotating.

The Lagrangian for a test particle traveling on the static space time $(\omega=0)$ described by (1) is given by:

$$
2 \mathcal{L}=-e^{2 \psi} \dot{t}^{2}+e^{-2 \psi}\left[e^{2 \gamma}\left(\dot{\rho}^{2}+\dot{z}^{2}\right)+\mu^{2} \dot{\varphi}^{2}\right]
$$

thus, the associated canonical momenta, $p_{x^{a}}=\frac{\partial \mathcal{L}}{\partial x^{a}}$, are:

$$
\begin{aligned}
p_{t}=-E & =-e^{2 \psi} \dot{t} \\
p_{\varphi}=L & =\mu^{2} e^{-2 \psi} \dot{\varphi} \\
p_{\rho} & =e^{-2(\psi-\gamma)} \dot{\rho} \\
p_{z} & =e^{-2(\psi-\gamma)} \dot{z}
\end{aligned}
$$

where $E$, and $L$, are constants of motion for each geodesic, a fact which comes from the symmetries of the space-time analyzed. As there is no explicit dependence on time, $t$, the Hamiltonian, $\mathcal{H}=p_{a} \dot{x}^{a}-\mathcal{L}$, is another conserved quantity, which we normalized to be equal to minus one half for time-like geodesics. Also, we restrict the motion to be at the equatorial plane, thus $\dot{z}=0$. In this way, we obtain the following equation for the radial geodesic motion:

$$
\dot{\rho}^{2}-e^{2(\psi-\gamma)}[E \dot{t}-L \dot{\varphi}-1]=0
$$

In order to have stable circular motion, which is the motion we are interested in, we have to satisfy three conditions:

i) $\dot{\rho}=0$, and

ii) $\frac{\partial V(\rho)}{\partial \rho}=0$, where $V(\rho)=-e^{2(\psi-\gamma)}[E \dot{t}-L \dot{\varphi}-1]$.

iii) $\left.\frac{\partial^{2} V(\rho)}{\partial \rho^{2}}\right|_{\text {extr }}>0$, in order to have a minimum.

With these conditions, from Eq.(7), we obtain a set of two equations constraining the motion to be circular extrema in the equatorial plane:

$$
\begin{aligned}
E \dot{t}-L \dot{\varphi}-1 & =0 \\
\frac{\partial}{\partial \rho}\left(e^{2(\psi-\gamma)}[E \dot{t}-L \dot{\varphi}-1]\right) & =0 .
\end{aligned}
$$

¿From Eq.(6), we can express $\dot{t}$, and $\dot{\varphi}$ in terms of $E, L$, and the metric coefficients as

$$
\begin{aligned}
\dot{t} & =e^{-2 \psi} E, \\
\dot{\varphi} & =\frac{e^{2 \psi}}{\mu^{2}} L .
\end{aligned}
$$

Using these equations in the constraints ones and recalling that $E$ and $L$ are constants for each circular orbit, after some rearranging, we arrive to the following equations:

$$
\begin{aligned}
& \mu^{2} e^{-2 \psi}\left(1-e^{-2 \psi} E^{2}\right)+L^{2}=0 \\
& -\left(e^{-2 \psi}\right)_{\rho} E^{2}+\left(\frac{e^{2 \psi}}{\mu^{2}}\right)_{\rho} L^{2}=0
\end{aligned}
$$


where the subindex stands for derivative with respect to $\rho$. Solving for $E$ and $L$, we obtain:

$$
\begin{aligned}
& E=e^{\psi} \sqrt{\frac{\frac{\mu_{\rho}}{\mu}-\psi_{\rho}}{\mu}-2 \psi_{\rho}}, \\
& L=\mu e^{-\psi} \sqrt{\frac{\psi_{\rho}}{\frac{\mu_{\rho}}{\mu}-2 \psi_{\rho}}} .
\end{aligned}
$$

The second derivative of the potential $V(\rho)$ evaluated at the extreme, in this case means evaluate at the values of $E$ and $L$ which constraint the motion to be circular and extrema, is given by:

$$
\left.V_{\rho \rho}\right|_{\text {extr }}=\frac{2 e^{2(\psi-\gamma)}}{\frac{\mu_{\rho}}{\mu}-2 \psi_{\rho}}\left(\frac{\mu_{\rho}}{\mu} \psi_{\rho \rho}-\frac{\mu_{\rho \rho}}{\mu} \psi_{\rho}+4 \psi_{\rho}^{3}-6 \frac{\mu_{\rho}}{\mu} \psi_{\rho}^{2}+3\left(\frac{\mu_{\rho}}{\mu}\right)^{2} \psi_{\rho}\right) .
$$

We can now obtain an expression for the angular velocity of a test particle, $\Omega$, moving in a circular motion in the orbital plane, in terms of the metric coefficients, recalling that

$$
\Omega=\frac{d \varphi}{d t}=\frac{\dot{\varphi}}{\dot{t}}
$$

thus, using Eqs.100, and (13), in this last equation for the angular velocity, we obtain that:

$$
\Omega=\frac{e^{2 \psi}}{\mu} \sqrt{\frac{\psi_{\rho}}{\mu}-\psi_{\rho}} .
$$

Finally, in order to express the tangential velocity of the test particles in circular motion in the equatorial plane, in terms of the metric coefficients, following Chandrasekhar [14], we rewrite the line element given in Eq.(1) as:

$$
d s^{2}=-e^{2 \psi} d t^{2}+e^{-2 \psi} \mu^{2} d \varphi^{2}+e^{-2(\psi-\gamma)}\left(d \rho^{2}+d z^{2}\right),
$$

thus, in terms of the proper time, $d \tau^{2}=-d s^{2}$, we have that

$$
\begin{aligned}
d \tau^{2}= & e^{2 \psi} d t^{2}\left[1-e^{-4 \psi} \mu^{2}\left(\frac{d \varphi}{d t}\right)^{2}+\right. \\
& \left.-e^{2 \gamma} e^{-4 \psi}\left(\left(\frac{d \rho}{d t}\right)^{2}+\left(\frac{d z}{d t}\right)^{2}\right)\right]
\end{aligned}
$$

from which we can write that

$$
1=e^{2 \psi} u^{0^{2}}\left[1-v^{2}\right],
$$

where $u^{0}=\frac{d t}{d \tau}$ is the usual time component of the four velocity, and a definition of the spatial velocity, $v^{2}$, comes out naturally in this way.

$$
v^{2}=e^{-4 \psi} \mu^{2}\left(\frac{d \varphi}{d t}\right)^{2}+e^{2 \gamma} e^{-4 \psi}\left(\left(\frac{d \rho}{d t}\right)^{2}+\left(\frac{d z}{d t}\right)^{2}\right)
$$

This spatial velocity is the 3-velocity of a particle measured with respect to an orthonormal reference system (see section 52 of [14]), thus has components:

$$
v^{2}=v^{(\varphi)^{2}}+v^{(\rho)^{2}}+v^{(z)^{2}} .
$$

¿From these last two expressions we obtain for the $\varphi$-component the spatial velocity:

$$
v^{(\varphi)}=e^{-2 \psi} \mu \Omega
$$


and substituting $\Omega$ from Eq. (16), we finally obtain an expression for the tangential velocity of a test particle in stable circular motion, in terms of the metric coefficients of the general line element given by Eq.(1), such tangential velocity has the form:

$$
v^{(\varphi)}=\sqrt{\frac{\psi_{\rho}}{\frac{\mu_{\rho}}{\mu}-\psi_{\rho}}} .
$$

It was our goal to obtain this expression for the tangential velocity for a general axisymmetric static space time, and to be able to describe it in terms of the metric coefficients alone, because now we can impose conditions on this tangential velocity, and deduce a constraint equation among the metric coefficients, which has to be satisfied in order to fulfill the condition imposed on the velocity. In particular, the tangential velocity for circular trajectories in each orbit is constant, that is $v_{\rho}^{(\varphi)}=0$, thus $v^{(\varphi)}=v_{c}^{(\varphi)}$, with $v_{c}^{(\varphi)}$ a constant, representing the value of the velocity, from Eq. (23), we have that:

$$
\frac{\mu_{\rho}}{\mu}=\frac{1+v_{c}^{(\varphi)^{2}}}{v_{c}^{(\varphi)^{2}}} \psi_{\rho}
$$

Finally, with respect to the $z$-motion, considering that at the equatorial plane not only $\dot{z}=0$, but also that $\ddot{z}=0$, that is that the forces above and below the plane cancel out, from the geodesic $z$-equation, using Eq.(24), we obtain that this relation among the metric coefficients must hold for the derivatives with respect to $z$ as well:

$$
\frac{\mu_{z}}{\mu}=\frac{1+v_{c}^{(\varphi)^{2}}}{v_{c}^{(\varphi)^{2}}} \psi_{z}
$$

In this way, we arrive to an if and only if condition: If Eqs.(24,25) is satisfied, then the tangential velocity of circular stable equatorial orbits is constant. Furthermore, if the tangential velocity of circular stable equatorial orbits is constant, then the metric coefficients have to satisfy Eqs.(24,25). Notice then that if the function $\psi$ and $\mu$ are related by

$$
e^{\psi}=\left(\frac{\mu}{\mu_{0}}\right)^{l}
$$

with $l=$ const, we obtain that this a necessary and sufficient condition for the velocity $v_{c}{ }^{(\varphi)}$ to be the same for two orbits at different radii at the equatorial plane, provided that $l=\left(v_{c}{ }^{(\varphi)}\right)^{2} /\left(1+\left(v_{c}^{(\varphi)}\right)^{2}\right)$.

We call your attention to the remarkable fact that the metric coefficient $\gamma$ does not play any role in this analysis, the motion analyzed is determined only by the other two metric coefficients, which now are related by this last equation, Eq.(25), thus leaving the problems in terms of only one metric coefficient. Actually this absence of $\gamma$ will be clear in the next section, where with the field equations we will see that it is determined in terms of the other metric coefficients and some components of the matter presented in the space-time, implying that it is not and independent function.

Thus, in order to have tangential velocities of equatorial objects circling the galaxy, and whose magnitude is radii independent, the form of the line element in the equatorial plane has to be

$$
d s^{2}=-\left(\frac{\mu}{\mu_{0}}\right)^{2 l} d t^{2}+\left(\frac{\mu}{\mu_{0}}\right)^{-2 l}\left[e^{2 \gamma} d \rho^{2}+\mu^{2} d \varphi^{2}\right] .
$$

Notice that this type of space time definitely can not be asymptotically flat. Neither it has the form of a space time related with a central black hole. What can be said is that this line element describes the region where the tangential velocity of the test particles is constant all over that region, and that it has to be joined in the interior and in the exterior regions with other types of space times if one wishes to have a central black hole, and that the influence of the middle region ends at some distance and thus has an asymptotically flat external region.

Taking into account the constraint between $\psi$ and $\mu$ given by Eq.(26), the energy, angular momentum, the rotational velocity, and the second derivative of the potential have the final expressions: 


$$
\begin{aligned}
E & =\frac{\left(\frac{\mu}{\mu_{0}}\right)^{l}}{\sqrt{l_{-}}}, \\
L & =\frac{\mu_{0} v_{c}^{(\varphi)}\left(\frac{\mu}{\mu_{0}}\right)^{-1 / l_{+}}}{\sqrt{l_{-}}}, \\
\Omega & =\frac{v_{c}^{(\varphi)}}{\mu_{0}}\left(\frac{\mu}{\mu_{0}}\right)^{-l_{-} / l_{+}}, \\
\left.\frac{\partial^{2} V(\rho)}{\partial \rho^{2}}\right|_{e x t r} & =2 e^{2(\psi-\gamma)} \frac{l_{+}-l_{-}}{l_{+}^{2}}\left(\frac{\mu_{\rho}}{\mu}\right)^{2} .
\end{aligned}
$$

being $l_{+}=1+\left(v_{c}^{(\varphi)}\right)^{2}$ and $l_{-}=1-\left(v_{c}^{(\varphi)}\right)^{2}$.

Notice that the second derivative of the potential at the extreme is always positive, thus the circular equatorial curves with constant tangential velocity are stable.

Before going to the field equations, we think it useful to present our derivations applied to the Schwarzschild case, thus testing the expressions while recovering the well known results. Starting with the line elements in spherical coordinates:

$$
d s^{2}=-\left(1-\frac{2 M}{r}\right) d t^{2}+\left(1-\frac{2 M}{r}\right)^{-1} d r^{2}+r^{2}\left(d \theta^{2}+\sin ^{2} \theta d \phi^{2}\right)
$$

we perform the coordinate transformation

$$
r=\sqrt{\rho^{2}+z^{2}}+\frac{M^{2}}{4 \sqrt{\rho^{2}+z^{2}}}+M, \theta=\tan ^{-1}\left(\frac{\rho}{z}\right),
$$

(the inverse transformation is $\rho=R \sin \theta, z=R \cos \theta$, with $R=\frac{1}{2}\left(r-M+\sqrt{r^{2}-2 M r}\right)$ ), to obtain the line element in the Papapetrou form, Eq.(11), with $e^{2 \psi}=\left(\frac{\sqrt{\rho^{2}+z^{2}}-\frac{M}{2}}{\sqrt{\rho^{2}+z^{2}}+\frac{M}{2}}\right)^{2}, e^{2 \gamma}=\left(1-\frac{M^{2}}{4\left(\rho^{2}+z^{2}\right)}\right)^{2}, \mu=\rho\left(1-\frac{M^{2}}{4\left(\rho^{2}+z^{2}\right)}\right), \omega=0$. The horizon in this coordinates is located at $\sqrt{\rho^{2}+z^{2}}=\frac{M}{2}$.

Restricting the expressions to the equatorial plane, $z=0$, we have that $\left.\psi\right|_{z=0}=\ln \left(\left(\rho-\frac{M}{2}\right) /\left(\rho+\frac{M}{2}\right)\right),\left.\mu\right|_{z=0}=$ $\rho\left(1-M^{2} / 4 \rho^{2}\right)$, thus from Eqs. 13], we obtain, for the energy and angular momentum:

$$
E=\frac{\left(\rho-\frac{M}{2}\right)^{2}}{\left(\rho+\frac{M}{2}\right) \sqrt{\rho^{2}-2 M \rho+\frac{M^{2}}{4}}}, \quad L=\left(\rho+\frac{M}{2}\right)^{2} \sqrt{\frac{M}{\rho\left(\rho^{2}-2 M \rho+\frac{M^{2}}{4}\right)}},
$$

for the angular velocity test particles, from Eq.(16):

$$
\Omega=\sqrt{M} \rho^{-\frac{3}{2}}\left(1+\frac{M}{2 \rho}\right)^{-3},
$$

which gives us the Kepler law.. For the tangential velocity, from Eq.23) we obtain

$$
v^{(\varphi)}=\sqrt{\frac{M}{\rho}}\left(1-\frac{M}{2 \rho}\right)^{-1}
$$

with the known dependence as the inverse of the square root of the distance. Transforming back to spherical coordinates, it can be seen that our expressions agree with the usual ones, see for instance 15. For the second derivative of the potential, it is useful to write $\rho$ in terms of the horizon radius, as $\rho=n \frac{M}{2}$, with $n$ a number, and from Eq.(14) we obtain:

$$
\left.V_{\rho \rho}\right|_{e x t r}=\frac{4 e^{2(\psi-\gamma)}}{M^{2} n(n+1)^{3}} \frac{\left(n^{2}-10 n+1\right)}{\left(n^{2}-4 n+1\right)}
$$


This second derivative is positive down to $\rho=(5+2 \sqrt{6}) \frac{M}{2}$, which marks the last stable orbit, and corresponds to the known result of $r=6 \mathrm{M}$ in spherical coordinates.

In this way, we are confident on our expressions and can proceed to study the field equations.

A last remark about the geometric analysis. Recall that the observations are based on measurements of the red shift, not on the tangential velocity directly. If the space time is flat, the two quantities are proportional. But we are now working in curved space times, so we have to see if that proportionality is still valid. Following [16], we use the fact that the frequency of a photon is given by $\nu=u^{\alpha} p_{\alpha}$, with $u^{\alpha}$ the four velocity of the object and $p^{\alpha}$ the photon momentum, we have that the red shift, $z$, is given by

$$
z=1-\frac{\nu_{e m}}{\nu_{r e c}}
$$

thus, for an object orbiting the galactic center in the equatorial plane at a distance $\rho$ from the center, with tangential velocity $v^{(\varphi)}$ and emitting a photon with frequency $\nu_{0}$, and for an observer located at rest at infinity, that is far away from the emission, detecting the photon with a frequency $\nu_{\infty}$, it can be shown that the red shift is given by:

$$
z=1-\frac{\left(1+v^{(\varphi)}\right)}{\sqrt{1-v^{(\varphi)}}} \sqrt{\frac{g_{t t}(\rho)}{g_{t t}(\infty)}} .
$$

Now, for the observed velocities, we have that $v \ll 1, i$. e. they are much less than the speed of light, and we have to suppose that far away from the observed galaxy its gravitational influence ends, otherwise we could not detect the tangential velocity, we would then be moving along with the observed object! Thus we can take $g_{t t}(\infty)=-1$ and we have that at first order in the velocity, with $g_{t t}(\rho)=-e^{\psi}=-1-\psi-\cdots$, we obtain:

$$
z=-\left(v^{(\varphi)}+\psi+\cdots\right) .
$$

But we have computed that in the case analyzed $\psi=v^{(\varphi)^{2}} /\left(1+v^{(\varphi)^{2}}\right) \ln \left(\mu / \mu_{0}\right) \sim 2 v^{(\varphi)^{2}}(\mu-1) /(\mu+1)$, thus $\psi \sim v^{(\varphi)^{2}}$, and we conclude that $z \sim v^{(\varphi)}$. In this way, we see that the radii independence of the value of the measured red shift can be related with the radii independence of the value of the tangential velocity, which is the fact that has been studied in this work.

\section{FIELD EQUATIONS}

Now we are in a position where we can test any type of matter-energy to determine whether or not it produces a curvature in the space time such that the motion of the test particles can be circular stable and be such that the tangential velocity of those particles is constant for a large radial region in the equatorial plane.

We obtain the general form of the Einstein tensor for the axisymmetric static space time described by Eq. (1), with $\omega=0$, and equate it to an arbitrary stress energy tensor. After some manipulations we conclude that the field equations are a set of two equations involving the metric coefficients $\psi$, and $\mu$ :

$$
\begin{aligned}
& \mu\left(\psi_{\rho \rho}+\psi_{z z}\right)+\mu_{\rho} \psi_{\rho}+\mu_{z} \psi_{z}=4 \pi \mu\left[e^{-2(\psi-\gamma)}\left(e^{-2 \psi} T_{t t}+\frac{e^{2 \psi}}{\mu^{2}} T_{\varphi \varphi}\right)+T_{\rho \rho}+T_{z z}\right], \\
& \mu_{\rho \rho}+\mu_{z z}=8 \pi \mu\left[T_{\rho \rho}+T_{z z}\right] .
\end{aligned}
$$

There are also two first order equations for the other metric coefficient $\gamma$ :

$$
\begin{aligned}
& \gamma_{\rho} \mu_{\rho}-\gamma_{z} \mu_{z}-\mu\left(\psi_{\rho}^{2}-\psi_{z}^{2}\right)+\mu_{z z}=8 \pi \mu T_{\rho \rho}, \\
& \gamma_{\rho} \mu_{z}+\gamma_{z} \mu_{\rho}-2 \mu \psi_{\rho} \psi_{z}-\mu_{\rho z}=8 \pi \mu T_{\rho z},
\end{aligned}
$$

and finally, the field equations give us another equation for the second derivatives of $\gamma$ which thus is redundant, this equation is:

$$
\gamma_{\rho \rho}+\gamma_{z z}+\left(\psi_{\rho}\right)^{2}+\left(\psi_{z}\right)^{2}=8 \pi \frac{e^{2 \gamma}}{\mu^{2}} T_{\varphi \varphi} .
$$


The analysis presented in the last section is exact and the relations between the metric coefficients and their first derivatives must be satisfied at the equatorial plane in order to describe the observed motion. Using the Einstein's equations, we need the second derivatives of those metric coefficients. Thus, we have to make the approximation that the relations obtained among them, holds as well in a region close to the equatorial plane. Within this approximation, from Eqs. 24, 25,26), it can be obtained the following expression:

$$
\mu\left(\psi_{\rho \rho}+\psi_{z z}\right)+\mu_{\rho} \psi_{\rho}+\mu_{z} \psi_{z}=\left(v_{c}{ }^{(\varphi)}\right)^{2} /\left(1+\left(v_{c}{ }^{(\varphi)}\right)^{2}\right)\left(\mu_{\rho \rho}+\mu_{z z}\right) .
$$

Thus, with this last relation, from the Einstein's equations Eqs. (41,42) we obtain a constraint among the stress energy tensor components which, within the approximation made on the validity of Eqs. 24, 25, 26) out of the equatorial plane, has to be satisfied by any type of matter in order to have constant tangential velocities:

$$
-\left(\frac{1-\left(v_{c}^{(\varphi)}\right)^{2}}{1+\left(v_{c}^{(\varphi)}\right)^{2}}\right)\left(T_{\rho \rho}+T_{z z}\right)=e^{-2(\psi-\gamma)}\left(e^{-2 \psi} T_{t t}+\frac{e^{2 \psi}}{\mu^{2}} T_{\varphi \varphi}\right)
$$

We finish our analysis by testing several types of matter, described by their respective stress energy tensor, to see whether or not they are able to deform the geometry of the space time in such a way that the tangential velocity of the equatorial rotational objects be constant, that is, that they satisfy Eq.(47).

\section{A. Vacuum Fields}

We start with the vacuum solutions, with $T_{\mu \nu}=0$. In this case Eq.47) is trivially satisfied, thus we proceed to analyze the Einstein equations directly. From Eq.(42), the easiest solution implies $\mu=\rho$. Eq.(41), is a Laplace equation for $\psi$ and 'imposing the condition that at the equatorial plane the flat curve condition, Eq.(25), be satisfied, as well as the one of symmetry with respect to the galactic plane, we obtain that $\psi=l \ln \rho$ and the other Einstein equation then imply $\gamma=l^{2} \ln \rho$, with $l=\left(v_{c}{ }^{(\varphi)}\right)^{2} /\left(1+\left(v_{c}{ }^{(\varphi)}\right)^{2}\right)$. In this way, we obtained an exact vacuum solution for the Einstein equations, which produces that the test particles circling at the equatorial plane behave in agreement with the observations:

$$
d s^{2}=-\rho^{2 l} d t^{2}+\rho^{-2 l}\left(\rho^{2 l^{2}}\left(d \rho^{2}+d z^{2}\right)+\rho^{2} d \varphi^{2}\right) .
$$

The central object is string-like. Observations show that cosmic strings object are very unlikely to exist, nevertheless, this is an example of objects which could produce the observed motion of test particles, and in which the density does not go as $r^{-2}$, because it is vacuum. Thus, such a behavior on the density is a sufficient but not a necessary condition for the flatness of the rotational curves.

\section{B. Perfect Fluid}

For the perfect fluid, $T_{\mu \nu}=(d+p) u_{\mu} u_{\nu}+g_{\mu \nu} p$, with $d$ the density of the fluid and $p$ its pressure. In this case we are thinking on a "dark fluid", which could be composed of planetoids or WIMPS or MACHOS, which are not seen but it is thought that they could be there affecting the geometry in the way needed in order to have the observed behavior in the tangential velocities of the luminous matter. Taking this dark fluid as static, the four velocity is given by $u^{\alpha}=\left(u^{0}, 0,0,0\right)$ with, for the line element given by Eq.(11) with $\omega=0, u^{0}=E e^{-2 \psi}$, and $L=0$. Thus $u_{0}=-E$, and from $u_{\alpha} u^{\alpha}=-1$, we conclude that $E=e^{\psi}$. The stress energy tensor for the dark fluid then has the form:

$$
\begin{aligned}
& T_{t t}=d E^{2}=d e^{2 \psi} \\
& T_{\rho \rho}=T_{z z}=e^{-2(\psi-\gamma)} p, \\
& T_{\varphi \varphi}=\mu^{2} e^{-2 \psi} p .
\end{aligned}
$$

Substituting in Eq.(47), we obtain that in the equatorial plane, in order to satisfy the observed behavior on the tangential velocities, the "dark fluid" has to satisfy:

$$
-2\left(\frac{1-\left(v_{c}^{(\varphi)}\right)^{2}}{1+\left(v_{c}^{(\varphi)}\right)^{2}}\right) p=(d+p)
$$


Thus, we obtain an equation of state for the "dark fluid" particles at the equatorial plane:

$$
p=-\frac{1+\left(v_{c}^{(\varphi)}\right)^{2}}{3-\left(v_{c}^{(\varphi)}\right)^{2}} d
$$

which, compared to the equation of state for a perfect fluid, $p=\omega d$, implies that $-1<\omega<-\frac{1}{3}$, for $v_{c}(\varphi)$ between the speed of light and zero. This result is quite remarkable. It coincides with the type of equation of state derived within the Quintessence model [17 20] at the cosmological level, and now we obtain similar results at the galactic level. This sort of matter has been called exotic matter [21] and studied in several contexts [22]. Our result points to the fact that the Dark Matter actually could be exotic. We want to stress that due to the approximation taken for the behavior of the metric coefficients off the galactic plane, we are not excluding the possibility that the dark fluid be composed of baryonic usual matter, actually from the Newtonian approach, we know that regular matter can produce the observed motion. This fact is not reproduced in the present analysis, due to our approximation, what we certainly can conclude is that exotic type of matter also can produce such 'observed motion.

In order to recover the Newtonian case, where we know that the dust type fluid does work as the dark matter, we have to analyze the spherical case, which is introduced in Appendix $\mathrm{V}$. For the stress energy tensor we again take the static perfect fluid, thus the four velocity of the test particle reads $u^{\mu}=\left(u^{0}, 0,0,0\right)$, with $u^{0}=\dot{t}$. For the spherically symmetric metric, Eq.(68), we know that $\dot{t}$ is associated to a conserved quantity, the energy, and is given by: $\dot{t}=\frac{E}{B(r)}$, and from the normalization of the four velocity, $u_{\alpha} u^{\alpha}=-1$, we get that $E=\sqrt{B(r)}$. Thus, considering those spacetimes for which the tangential velocity $\left(v_{c}\right)$ of test particles in circular orbits is radii independent, we use Eq. (76) , obtaining that $u^{0}=\frac{1}{\sqrt{B_{0}}} r^{-\left(v_{c}\right)^{2}}$, thus, $u_{0}=-\sqrt{B_{0}} r^{\left(v_{c}\right)^{2}}$. In this way, we get that the non zero components of the static spherically symmetric perfect fluid are:

$$
\begin{aligned}
T_{t t} & =d B_{0} r^{2\left(v_{c}\right)^{2}}, \\
T_{r r} & =p A(r), \\
T_{\theta \theta} & =p r^{2}
\end{aligned}
$$

Substituting these expressions in the Einstein equations Eq.(77), it turns out that the equations can be completely solved, yielding:

$$
\begin{aligned}
A(r) & =\frac{b}{2\left(1-A_{0} r^{\frac{b}{a}}\right)}, \\
d & =\frac{(2+a) A(r)-a-b}{8 \pi a r^{2} A(r)}, \\
p & =\frac{1+2\left(v_{c}\right)^{2}-A(r)}{8 \pi r^{2} A(r)},
\end{aligned}
$$

with $b=2\left(1+2\left(v_{c}\right)^{2}-v_{c}{ }^{4}\right), a=1+\left(v_{c}\right)^{2}$, and $A_{0}$ an integration constant.

Taking the particular case for the integration constant $A_{0}=0$, we get

$$
\begin{aligned}
A(r) & =\frac{b}{2}, \\
d & =\frac{\left(v_{c}\right)^{2}\left(1-\frac{\left(v_{c}\right)^{2}}{2}\right)}{2 \pi b r^{2}}, \\
p & =\frac{\left(v_{c}\right)^{4}}{4 \pi b r^{2}} .
\end{aligned}
$$

In this way, we obtain the particular solution where the particles move in the observed way, in a space-time with a deficit angle, $g_{r r}=$ constant, and in which the density goes as $d \sim\left(v_{c}\right)^{2} / r^{2}$, and the pressure goes as $p \sim\left(v_{c}\right)^{4} / r^{2}$, thus it is a dust like solution. Furthermore, for an equation of state $p=\omega d$, this dust like solution implies $\omega=\left(v_{c}\right)^{2} /\left(2\left(1-\left(v_{c}\right)^{2} / 2\right)\right)$, which is between 0 and 1 , for the tangential velocity between 0 and 1 , and thus is a perfectly well known fluid type. In this way we recover the dust hypotheses within our approach, and we clearly see that the' fact that we did not recover this case within the axi-symmetric analysis, was due to our approximation 
outside the equatorial plane.

For the general case when the integration constant $A_{0}$ is non zero, taking an equation of state as before $p=\omega d$, we obtain that the $\omega$ is a function of $r$ and again, as in the axial case, it is negative, so we are dealing with the exotic type of perfect fluid obtained in the axi-symmetric case.

This is a good moment to discuss the question on the need to use GR, even though the gravitational field is weak. In the Newtonian description it is well known that the space-time can be described as

$$
d s^{2}=-(1+2 \Phi) d t^{2}+(1-2 \Phi) d r^{2}+r^{2} d \Omega^{2}
$$

with $\Phi=\Phi(r)$ the Newtonian gravitational potential. For the spherically symmetric case we obtain that

$$
-g_{t t}=r^{2\left(v_{c}\right)^{2}}=e^{2\left(v_{c}\right)^{2} \ln (r)}=1+2\left(v_{c}\right)^{2} \ln (r)+\cdots
$$

from here we determine that $\Phi=\left(v_{c}\right)^{2} \ln (r)$. In this Newtonian approximation the complete set of 10 Einstein equations reduces to one equation, the usual Poisson equation $\nabla^{2} \Phi=4 \pi G d$. Collecting this last result, it is obtain that the density, $d$, goes as $d \sim\left(v_{c}\right)^{2} / r^{2}$, which is the expression for the dark matter density known from the astronomers' work.

However, notice that in this approximation it can not be said anything else about the matter producing the observed motion. The Newtonian approximation fixes the matter to be dust-perfect-fluid-like type. This is the usual way of reasoning: it is suppose a priori that the dark matter is a completely Newtonian dust and at the end of the day one arrives to a consistent description of the dark matter determining only the shape of the Newtonian gravitational potential.

In this work we are proceeding in a different way. We are using Einstein equations backwards; we do not make any assumptions in the type of matter nor do approximations. From the observations on the motion of the test particles, we determine the geometry and then, by means of the Einstein's equations we obtain constrains of the type of matter. We have shown that we do recover the Newtonian result, but also it is clear that this is a very particular case for a very specific type of matter. In the general reasoning, we do not fix neither the type of matter nor the equation of state, we let the equations themselves to do that obtaining more general results.

To end this argumentation we recall the reader that there is three conditions that have to be fulfill in order to reach the Newtonian limit; 1) the speeds of the study particles most be most less than the speed of light, 2) the gravitational field must be weak, and 3) the pressures associated with the matter study must to be must smaller than the corresponding density. It is this last condition which is taking a priori in the usual analysis and it is not satisfy in the general case as we have shown, thus justify the need of GR in order to be able to consider any type of matter.

As a last remark about these results on the type of perfect fluid is that the Big-Bang nucleosynthesis imposes very strong constraints to the percentage of the baryonic matter to the total content of the Universe. If the dark matter would be a dark fluid of baryonic matter, such percentage would be quite above the value settled by those constraints. Thus, even if the baryonic dark fluid can not be discarded by dynamical methods, the cosmological constraints make it unlikely, a fact which might strength the case for exotic type of dark fluids or for other type of dark matter.

\section{Cosmological Constant}

For a cosmological constant, $\Lambda, T_{\mu \nu}=\Lambda g_{\mu \nu}$. We thus have $T_{t t}=-e^{2 \psi} \Lambda, T_{\rho \rho}=T_{z z}=e^{-2(\psi-\gamma)} \Lambda, T_{\varphi \varphi}=e^{-2 \psi} \mu^{2} \Lambda$, thus from Eq.(47), we obtain

$$
\left(\frac{1-\left(v_{c}^{(\varphi)}\right)^{2}}{1+\left(v_{c}^{(\varphi)}\right)^{2}}\right) \Lambda=0
$$

In this way we see that, within our approximation, a non zero cosmological constant can not explain the observed behavior because implies that the observed tangential velocity had to be equal to $1, i$. e., they should be moving at the speed of light. Something similar occurs with the scalar field. 


\section{Scalar Field}

For scalar field $\phi$ with potential, $T_{\mu \nu}=\phi_{, \mu} \phi_{, \nu}-\frac{1}{2} g_{\mu \nu} \phi^{\alpha} \phi_{\alpha}+g_{\mu \nu} V(\phi)$. We have that, due to the symmetry of our space time $\phi=\phi(\rho, z)$ the $T_{\mu \nu}$ components are:

$$
\begin{aligned}
T_{t t} & =\frac{1}{2} e^{2(2 \psi-\gamma)}\left(\phi_{\rho}{ }^{2}+\phi_{z}{ }^{2}\right)-e^{2 \psi} V(\phi), \\
T_{\rho \rho} & =\frac{1}{2}\left(\phi_{\rho}{ }^{2}-\phi_{z}{ }^{2}\right)+e^{-2(\psi-\gamma)} V(\phi), \\
T_{z z} & =-\frac{1}{2}\left(\phi_{\rho}{ }^{2}-\phi_{z}{ }^{2}\right)+e^{-2(\psi-\gamma)} V(\phi), \\
T_{\rho z} & =\phi_{\rho} \phi_{z}, \\
T_{\varphi \varphi} & =-\frac{1}{2} e^{-2 \gamma} \mu^{2}\left(\phi_{\rho}{ }^{2}+\phi_{z}{ }^{2}\right)+e^{-2 \psi} \mu^{2} V(\phi) .
\end{aligned}
$$

Inserting these components in Eq.(47), we obtain that, as in the cosmological constant case

$$
\left(\frac{1-\left(v_{c}^{(\varphi)}\right)^{2}}{1+\left(v_{c}^{(\varphi)}\right)^{2}}\right) V(\phi)=0
$$

Again, either the particles move at the speed of light, or the scalar field potential is zero at the equatorial plane. When the scalar field potential is zero, that is, we have a massless scalar field, Eq.(47) is satisfied, and we have to go back to the Einstein's equations as in the vacuum case. Again from Eq.(42), we take the simplest solution $\mu=\rho$, and, as in the vacuum case, we obtain $\psi=l \ln \rho$. The last metric coefficient $\gamma$, can be solved in terms of the scalar field. Thus, to have a complete solution, it is only left to solve the Klein Gordon equation for the scalar field: $D^{2} \phi+\frac{1}{\mu} D \phi D \mu=0$, which turns out to be the same equation for the metric coefficient $\psi$. However, in this case we do not have boundary conditions well defined: The space time is not asymptotically flat; it is not known the form of the space time near and at the origin, we are only analyzing the region where the curves are flat; there are no conditions for the scalar field at the equatorial plane. What can be concluded at this stage, is that the scalar field does remain being a candidate for the dark matter, and thus to contribute with about $25 \%$ of the matter of the Universe.

\section{CONCLUSIONS}

We have found that in a static, axisymmetric space-time, a sufficient and necessary condition in order to have a flat profile for the rotational curves in a plane of that space-time is that its metric tensor must have the form given by Eq. (27). This form of the metric must be the one required for galaxies in the region where the rotational curves profile of stars is flat. It is important to stress the fact that in the derivation of this expression, only the geometry of the space-time was involved, thus, it is independent of the type of matter which generates such a geometry; that is, whatever the matter might be, Eq. (27) must be the form of the line element at the galactic plane for a static axisymmetric space time which can be expressed in the Papapetrou form, Eq. (11) with $\omega=0$, thus, this result is not only the general relativistic analog of the Newtonian result for the gravitational Newtonian potential, $\phi(r) \sim 1 / r$, but it can be used for any type of matter, including those which do not have a clear Newtonian expression, such as the scalar field.

With this idea in mind, we proceeded further using the Einstein's equations, which essentially describe the inter dependence of matter-energy and geometry. We had to accept some loose of the generality of our results, in making the assumption that the definite relation which we obtained for the metric coefficients at the galactic plane, Eqs. (26), are also valid in a close by region off the plane, and thus determine a relation for the second derivatives of the metric coefficients, Eq.(46). Within this approximation, we were able to obtain a constraint equation among the components of a general stress energy tensor, $T^{\alpha \beta}$, Eq.(47). We tested this expression in four types of stress energy tensors which included the traditional types of matter which have been used as candidates for the dark matter in the galactic halos, such as the perfect fluid or the cosmological constant. We obtained that for the vacuum case, a cosmic string type of matter does generates the observed motion of test particles. Even though the cosmic strings are unlikely objects to be the Universe, it was a clear example for the fact that the Newtonian behavior of the density, $\rho(r) \sim 1 / r^{2}$, is not a necessary condition for describing the observed motion. We analyzed also the static perfect fluid, and it is interesting 
that in difference with the Newtonian description, within the general relativistic formulation, we are able to obtain conditions for the equation of state of the dark fluid. Even though we were not able to reproduce the well known Newtonian result for dust-like fluid, due to our approximation for the second derivatives of the metric coefficients, we did showed that a dark fluid with an exotic type of matter, is a candidate for being the dark matter. Furthermore, this results represents the Quintessence type of matter at a galactic level. We performed the study in the spherically symmetric static space time in appendix $\mathrm{V}$, and when applied to the perfect fluid static case we did recover the Newtonian result, namely that the "dark fluid" could be a well behaved dust like fluid. We further analyzed the cosmological constant case, which within our approximation implied that it can not be, and the massive scalar field case, which again, within our approximation, turned out that it has to be massless, and the massless scalar field also remains being a candidate for the dark matter.

As we have mentioned above, our results are useful for describing the region where is observed the flat behavior of the test particles rotating around the galactic center, it is clearly needed to proceed further in order to be able to describe the motion in the complete region, from the center to the exterior. Some preliminary results indicate that a combination of perfect fluid with baryonic matter and some of the matter analyzed here, could be in the right direction, 12]. Also, the approximation we made for the second derivatives of the metric coefficients, has to be further analyzed. It would be of great help, in order to be able to apply the present description in objets out from the glactic plane, to have a sample of the profile of velocities of such objects. In the simpler spherically symmetric case, there is no need to make such approximation, and more definite results can be obtained, though with more restrictions on the geometry. Besides, there are reasons to belive that the dark matter halo is spherical [23], thus it might be a good approximation the analysis made within this symmetry. We studied the scalar field in this symmetry in [24].

Finally, as the best way to study dark matter is through its effects on the dynamics of the visible objetcs, further studies along the lines presetned in this work can be performed using gravitational lensing or jets.

Overall, we consider that the analysis presented in this work, is on the right track in order to determine which is the type of matter which constitutes the $90 \%$ of the matter in the galaxies.

\section{ACKNOWLEDGMENTS}

This work was partially supported by a grant CONACyT-DFG, by CONACyT, México, under grants 94890 (F.S.G.), and DGAPA-UNAM IN121298 (D.N.) We want to thank the relativity group in Jena for its kind hospitality and partial support.

In this appendix we present an analogous derivation for the conditions which the constancy of the tangential velocity of circular orbits impose on the metric coefficients for the spherical static case. Furthermore, we also present the Einstein equations in this case for a general stress energy tensor.

It is interesting that, due to the symmetries, in this case we do not have to restrict the analysis to equatorial orbits, and that the above mentioned conditions give a closed form for the metric coefficient $g_{t t}$.

We begin with the line element

$$
d s^{2}=-B(r) d t^{2}+A(r) d r^{2}+r^{2}\left(d \theta^{2}+\sin ^{2} \theta d \varphi^{2}\right)
$$

The Lagrangian for a test particle reads

$$
2 \mathcal{L}=-B(r) \dot{t}^{2}+A(r) \dot{r}^{2}+r^{2}\left(\dot{\theta}^{2}+\sin ^{2} \theta \dot{\varphi}^{2}\right) .
$$

We infer the conserved quantities, the energy $E=B(r) \dot{t}$, the $\varphi$-momentum $L_{\varphi}=r^{2} \sin ^{2} \theta \dot{\varphi}$, and the total angular momentum, $L^{2}=L_{\theta}^{2}+\left(\frac{L_{\varphi}}{\sin \theta}\right)^{2}$, with $L_{\theta}=r^{2} \dot{\theta}$. The radial motion equation can thus be written as:

$$
\dot{r}^{2}+V(r)=0
$$

with the potential $V(r)$ given by

$$
V(r)=-\frac{1}{A(r)}\left(\frac{E^{2}}{B(r)}-\frac{L^{2}}{r^{2}}-1\right)
$$


Notice that, due to the spherical symmetry, we do not need to restrict the study to equatorial orbits, this last radial motion is valid for any angle $\theta$. For circular stable orbits, we again have the conditions, $\dot{r}=0, V_{r}=0$, and $V_{r r}>0$, which imply the following expressions for the energy and total momentum of the particles in such orbits:

$$
\begin{aligned}
E^{2} & =\frac{2 B(r)^{2}}{2 B(r)-r B(r)_{r}}, \\
L^{2} & =\frac{r^{3} B(r)_{r}}{2 B(r)-r B(r)_{r}},
\end{aligned}
$$

and for the second derivative of the potential evaluated at the extrema

$$
\left.V(r)_{r r}\right|_{\text {extr }}=2 \frac{\frac{r B(r)_{r r}}{B}+\frac{B(r)_{r}}{B}\left(3-\frac{2 r B(r)_{r}}{B}\right)}{r A(r)\left(2-\frac{r B(r)_{r}}{B}\right)} .
$$

On the other hand, in a similar manner as it was presented in the text, we obtain that the tangential velocity, $\left(v_{c}\right)^{2}=\frac{r^{2}}{B(r)}\left(\left(\frac{d \theta}{d t}\right)^{2}+\sin ^{2} \theta\left(\frac{d \varphi}{d t}\right)^{2}\right)$, for particles in stable circular orbits is given by:

$$
\left(v_{c}\right)^{2}=\frac{r B(r)_{r}}{2 B(r)}
$$

Thus, imposing the observed condition that this tangential velocity velocity is constant for several radii, this last equation can be integrated for the metric coefficient $g_{t t}$ :

$$
B(r)=B_{0} r^{2\left(v_{c}\right)^{2}}
$$

with $B_{0}$ an integration constant.

In this way, we again arrive to a theorem, stating that: For a static spherically symmetric spacetime, $v_{c}$, the tangential velocity of particles moving in circular stable orbits is radii independent if and only if the $g_{t t}$ metric coefficient has the form $g_{t t}=B_{0} r^{2\left(v_{c}\right)^{2}}$.

Notice that in this case, one of the metric coefficients was completely integrated and the other one, $A(r)$ remains arbitrary. Also, as mentioned, the analysis made no suppositions on the plane of motion, so the result is valid for any circular stable trajectory.

Finally, we can construct the Einstein tensor and arrive to the following Einstein equations which give us information about the type of matter curving the spacetime in such a way that the motion corresponds to the observed one:

$$
\begin{aligned}
\frac{B_{0} r^{2\left(v_{c}\right)^{2}}}{A(r)^{2}}\left(r A(r)^{\prime}+A(r)(A(r)-1)\right) & =8 \pi T_{t t}, \\
\frac{2\left(v_{c}\right)^{2}+1-A(r)}{r^{2}} & =8 \pi T_{r r}, \\
-\frac{r\left(\left(v_{c}\right)^{2}+1\right) A(r)^{\prime}-2 v_{c}^{4} A(r)}{2 A(r)^{2}} & =8 \pi T_{\theta \theta},
\end{aligned}
$$

where' ${ }^{\prime}$ stands for derivative with respect to $r$. This study was applied for the scalar field in [24].

In this appendix we present the generalization of the derivation of the constraint equation among the metric coefficients, Eq.(25), for the stationary case, where $\omega \neq 0$, described by the line element 1 .

¿From Eq.(6), we express $\dot{t}$, and $\dot{\varphi}$. in terms of $E, L$, and the metric coefficients as

$$
\begin{aligned}
\dot{t} & =\frac{e^{2 \psi}}{\mu^{2}}\left[\left(\mu^{2} e^{-4 \psi}-\omega^{2}\right) E-\omega L\right], \\
\dot{\varphi} & =\frac{e^{2 \psi}}{\mu^{2}}(\omega E+L) .
\end{aligned}
$$


Using these equation in the constraints ones, Eqs.(8) we arrive at:

$$
\begin{array}{r}
\mu^{2} e^{-2 \psi}\left(1-e^{-2 \psi} E^{2}\right)+(\omega E+L)^{2}=0, \\
-\left(e^{-2 \psi}\right)_{\rho} E^{2}+\left(\frac{e^{2 \psi}}{\mu^{2}}\right)_{\rho}(\omega E+L)^{2}+\frac{2 e^{2 \psi}}{\mu^{2}}(\omega E+L) \omega_{\rho} E=0,
\end{array}
$$

Solving for $E$ and $L$, we obtain:

$$
\begin{aligned}
& E=e^{\psi} \sqrt{\frac{\mathcal{A}}{\mathcal{B}}} \\
& L=\frac{\mu e^{-\psi}}{\sqrt{\mathcal{B}}}\left(\sqrt{\mathcal{A}-\mathcal{B}}-\frac{\omega e^{2 \psi}}{\mu} \sqrt{\mathcal{A}}\right),
\end{aligned}
$$

where

$$
\begin{aligned}
& \mathcal{A}= 2 e^{-4 \psi}\left(\mu_{\rho}-\mu \psi_{\rho}\right)\left(\mu_{\rho}-2 \mu \psi_{\rho}\right)-\left(\omega_{\rho}\right)^{2}+ \\
& \pm \omega_{\rho} \sqrt{\left(\omega_{\rho}\right)^{2}-4 \mu \psi_{\rho} e^{-4 \psi}\left(\mu_{\rho}-\mu \psi_{\rho}\right)} \\
& \mathcal{B}=2 e^{-4 \psi}\left(\mu_{\rho}-2 \mu \psi_{\rho}\right)^{2}-2\left(\omega_{\rho}\right)^{2} .
\end{aligned}
$$

For the second derivative of the potential $V(\rho)$ evaluated at the extreme, we obtain:

$$
\begin{aligned}
\left.V_{\rho \rho}\right|_{\text {extr }}= & -\frac{2 e^{2(\psi-\gamma)}}{\mathcal{B}}\left[\left(2 \psi_{\rho \rho}-\frac{\mu_{\rho \rho}}{\mu}+3\left(\frac{\mu_{\rho}}{\mu}\right)^{2}-4 \frac{\mu_{\rho}}{\mu} \psi_{\rho}+\frac{e^{4 \psi}}{\mu^{2}}\left(\omega_{\rho}\right)^{2}\right) \mathcal{A}\right. \\
& -\left(\psi_{\rho \rho}-\frac{\mu_{\rho \rho}}{\mu}+2\left(\psi_{\rho}\right)^{2}+3\left(\frac{\mu_{\rho}}{\mu}\right)^{2}-4 \frac{\mu_{\rho}}{\mu} \psi_{\rho}\right) \mathcal{B}+ \\
& \left.\left(4\left(\psi_{\rho}-\frac{\mu_{\rho}}{\mu}\right) \omega_{\rho}+\omega_{\rho \rho}\right) \frac{\sqrt{\mathcal{A}(\mathcal{A}-\mathcal{B})}}{\mu}\right] .
\end{aligned}
$$

On the other hand, using Eqs.(79), and (81), in the expression for the angular velocity, Eq.(15) we obtain that:

$$
\Omega=\frac{e^{2 \psi}}{\mu} \frac{\sqrt{\mathcal{A}-\mathcal{B}}}{\sqrt{\mathcal{A}}-\frac{\omega e^{2 \psi}}{\mu^{2}} \sqrt{\mathcal{A}-\mathcal{B}}},
$$

where $\mathcal{A}$ and $\mathcal{B}$ are given by Eqs. 83 .

As in the static case, Following Chandrasekhar [14], we rewrite the line element given in Eq.(11) as:

$$
\begin{aligned}
d s^{2}= & -\frac{\mu^{2} e^{-2 \psi}}{\mu^{2} e^{-4 \psi}-\omega^{2}} d t^{2}+e^{2 \psi}\left(\mu^{2} e^{-4 \psi}-\omega^{2}\right)\left(d \varphi-\frac{\omega}{\mu^{2} e^{-4 \psi}-\omega^{2}} d t\right)^{2}+ \\
& e^{-2(\psi-\gamma)}\left(d \rho^{2}+d z^{2}\right)
\end{aligned}
$$

thus, in terms of the proper time, $d \tau^{2}=-d s^{2}$, we have that

$$
\begin{aligned}
d \tau^{2}= & \frac{\mu^{2} e^{-2 \psi}}{\mu^{2} e^{-4 \psi}-\omega^{2}} d t^{2}\left[1-\frac{e^{4 \psi}\left(\mu^{2} e^{-4 \psi}-\omega^{2}\right)^{2}}{\mu^{2}}\left(\frac{d \varphi}{d t}-\frac{\omega}{\mu^{2} e^{-4 \psi}-\omega^{2}}\right)^{2}+\right. \\
& \left.-\frac{e^{2 \gamma}\left(\mu^{2} e^{-4 \psi}-\omega^{2}\right)}{\mu^{2}}\left(\left(\frac{d \rho}{d t}\right)^{2}+\left(\frac{d z}{d t}\right)^{2}\right)\right]
\end{aligned}
$$

from which we can write that

$$
1=\frac{\mu^{2} e^{-2 \psi}}{\mu^{2} e^{-4 \psi}-\omega^{2}} u^{0^{2}}\left[1-v^{2}\right]
$$


where $u^{0}=\frac{d t}{d \tau}$ is the usual time component of the four velocity, and a definition of the spatial velocity, $v^{2}$, again comes out naturally in this way.

$$
\begin{aligned}
v^{2}= & \frac{e^{4 \psi}\left(\mu^{2} e^{-4 \psi}-\omega^{2}\right)^{2}}{\mu^{2}}\left(\frac{d \varphi}{d t}-\frac{\omega}{\mu^{2} e^{-4 \psi}-\omega^{2}}\right)^{2}+ \\
& +\frac{e^{2 \gamma}\left(\mu^{2} e^{-4 \psi}-\omega^{2}\right)}{\mu^{2}}\left(\left(\frac{d \rho}{d t}\right)^{2}+\left(\frac{d z}{d t}\right)^{2}\right),
\end{aligned}
$$

which is the 3 -velocity of a particle measured with respect to an orthonormal reference system, it has components:

$$
v^{2}=v^{(\varphi)^{2}}+v^{(\rho)^{2}}+v^{(z)^{2}} .
$$

For the $\varphi$-component of the spatial velocity we obtain:

$$
v^{(\varphi)}=\frac{e^{2 \psi}}{\mu}\left[\left(\mu^{2} e^{-4 \psi}-\omega^{2}\right) \Omega-\omega\right],
$$

and substituting $\Omega$ from Eq. 85 , we finally obtain an expression for the tangential velocity of a test particle in stable circular motion:

$$
v^{(\varphi)}=\frac{\mu e^{-2 \psi} \sqrt{\mathcal{A}-\mathcal{B}}-\omega \sqrt{\mathcal{A}}}{\mu e^{-2 \psi} \sqrt{\mathcal{A}}-\omega \sqrt{\mathcal{A}-\mathcal{B}}}
$$

where $\mathcal{A}$ and $\mathcal{B}$ are given by Eqs.(83).

Imposing the condition of constancy for all radii, that is $v_{\rho}^{(\varphi)}=0$, thus $v^{(\varphi)}=v_{c}^{(\varphi)}$, with $v_{c}^{(\varphi)}$ a constant, representing the value of the velocity, from Eq. (92), we finally have that:

$$
\mathcal{B}=\left(1-v_{c}^{(\varphi)^{2}}\right) F^{2} \mathcal{A},
$$

where $F=\left(\mu^{2} e^{-4 \psi}-\omega^{2}\right) /\left(\mu e^{-2 \psi}+v_{c}^{(\varphi)} \omega\right)^{2}$. This last expression, represents a constraint among three of the metric coefficients, and we can express one of them, say $\omega$, in terms of the other two: $\psi$, and $\mu$. In this way, we again arrive to an iff condition, namely: The tangential velocity of a test particle moving in a circular equatorial motion in an axisymmetric stationary background, has a radii independent magnitude iff the metric coefficients satisfy the constraint equation (93).

[1] M. S. Turner, Astron. Soc. Pac. Conf. Series, Vol. 666, 1999. Preprint astro-ph/9811454.

[2] P. J. E. Peebles, "Principles of Physical Cosmology", Princeton University Press, (1993).

[3] P. de Bernardis et al., Nature 404 (2000) 955-959. S. Hanany et al., ApJ Letters 545 (2000) 5.

[4] D. N. Schramm, in "Nuclear and Particle Astrophysics", ed. J. G. Hirsch and D. Page, Cambridge Contemporary Astrophysics, (1998). X. Shi, D. N. Schramm and D. Dearborn, Phys. Rev. D 50 (1995) 2414-2420.

[5] G. Jungman et al. Phys Rep. 267 (1996) 195.

[6] M. Milgrom, ApJ 270 (1983) 365-370. M. Milgrom, ApJ 270 (1983) 371-383. M. Milgrom, ApJ 270 (1983) 384-389.

[7] P. D. Mannheim and D. Kazanas, ApJ 342 (1989) 635-638. P. D. Mannheim, ApJ 479 (1997) 659.

[8] J. F. Navarro, C. S. Frenk and S. D. M. White, MNRAS 462 (1996) 563. J. F. Navarro, C. S. Frenk and S. D. M. White, ApJ 490 (1997) 493.

[9] V. Avila-Reese, C. Firmani, A. Klypin and A. V. Kravtsov, MNRAS 309 (1999) 507.

[10] V. C. Rubin, N. Thonnard and W. K. Ford, ApJ 225 (1978) L107. V. C. Rubin, N. Thonnard, W. K. Ford, ApJ 238 (1980) 471. M.

[11] Persic, P. Salucci and F. Stel., MNRAS 281 (1996) 27-47.

[12] F. S. Guzmán and T. Matos, Class. Quantum Grav. 17 (2000) L9-L16. T. Matos and F. S. Guzman, Ann. Phys. (Leipzig) 9 (2000) SI-133. 
[13] Sir Arthur Conan Doyle, "The Annotated Sherlock Holmes, The adventure of the Beryl Coronet", p.299, Clarkson N. Potter Inc., New York (1967).

[14] S. Chandrasekhar, "Mathematical theory of black holes", Claredon Press Oxford (1983).

[15] A. P. Lightman, W. H. Press, R. H. Price, and S. A. Teukolsky, "Problem book in relativity and gravitation", Princeton University Press, problem 15.8, (1979).

[16] R. M. Wald, "General Relativity", The University of Chicago Press (1984).

[17] J. P. Ostriker, and P. J. Steinhardt, Nature 377 (1995) 600.

[18] R. R. Caldwell, Rahul Dave and Paul J. Steinhardt, Phys. Rev. Lett. 80 (1998) 1582-1585.

[19] Ivaylo Zlatev, Limin Wang and Paul J. Steinhardt, Phys. Rev. Lett. 82 (1988) 896-899.

[20] S. M. Carroll, Phys. Rev. Lett. 81 (1998) 3067-3070, C. Armendariz, V. Mukhanov, and P. J. Steinhardt, preprint astro$\mathrm{ph} / 0004134$.

[21] M. S. Morris and K. S. Thorne, Am. J. Phys. 56 (1988) 395-412.

[22] J. Goodman, to be published. Preprint astro-ph/0003018.

[23] R. Ibata, M. Irwin, G. Lewis and A. Stolte, ApJ Letters, in press. Preprint astro-ph/0004255.

[24] T. Matos, F. S. Guzmán and D. Núñez, Phys. Rev. D 62 (2000) 061301. 